\title{
Multidetector row CT for imaging the paediatric tracheobronchial tree
}

\author{
Georgia Papaioannou • Carolyn Young • \\ Catherine M. Owens
}

Received: 26 January 2007 / Accepted: 17 February 2007 / Published online: 25 April 2007

(C) Springer-Verlag 2007

\begin{abstract}
The introduction of multidetector row computed tomography (MDCT) scanners has altered the approach to imaging the paediatric thorax. In an environment where the rapid acquisition of $\mathrm{CT}$ data allows general hospitals to image children instead of referring them to specialist paediatric centres, it is vital that general radiologists have access to protocols appropriate for paediatric applications. Thus a dramatic reduction in the delivered radiation dose is ensured with optimal contrast bolus delivery and timing, and inappropriate repetition of the scans is avoided. This article focuses on the main principles of volumetric CT imaging that apply generically to all MDCT scanners. We describe the reconstruction techniques for imaging the paediatric thorax and the low-dose protocols used in our institution on a 16-slice detector CT scanner. Examples of the commonest clinical applications are also given.
\end{abstract}

Keywords Volumetric · Multidetector CT .

Tracheobronchial tree $\cdot$ Children

\section{Introduction}

Imaging of the airways with subsequent 2-D and 3-D reconstructions has significantly improved with the advent of the multidetector row computed tomography (MDCT) scanners. These scanners allow volumetric acquisition of

G. Papaioannou $(\bowtie) \cdot$ C. Young $\cdot$ C. M. Owens

Radiology Department,

Great Ormond Street Hospital for Children NHS Trust,

Great Ormond Street,

London WC1N 3JH, UK

e-mail: gpapaio@hotmail.com isotropic datasets. As a result, the scanning time is significantly reduced, and large anatomical regions are covered within seconds with optimal intravenous contrast material enhancement. MDCT scanners have significantly improved the image quality as the artefacts resulting from partial volume averaging and motion have been dramatically reduced. Additionally, their fast and user-friendly workstations allow postprocessing of impressive multiplanar 2-D and 3-D reconstructions, which are desirable to clinicians as they can be used for comprehensive presurgical planning $[1,2]$. In chest applications, the whole of the tracheobronchial tree can be adequately depicted with a single-breath-hold volume acquisition within 4-6 s; normal or complex anatomical structures, pathological processes and their anatomical relationships can be precisely imaged [3, 4]. Finally, on the 16-slice MDCT scanner, by applying the "Combiscan" protocol $(0.75-\mathrm{mm}$ collimated scans after administration of intravenous contrast medium), postprocessing of the original data pool can additionally generate high-resolution images of the chest.

\section{Technicalities for MDCT of the paediatric airways}

Imaging of the airways and thoracic vessels in infants and children has been significantly affected by the wide spectrum of applications of 16-slice MDCT. The key achievement with MDCT is the increased volume coverage per time unit at high axial resolution, which results in improved temporal resolution. With MDCT, scanning techniques may vary when different collimation is selected: the narrowest collimation is applied when partial volume effect needs to be minimal and the 3-D postprocessing images of optimal quality, e.g. CT angiography (CTA) and cardiac CT [3]. 
Preparation of the child: is sedation always needed?

Due to the faster scanning times possible with MDCT scanners, routine sedation is no longer required. In the neonate, recent feeding usually provides tranquillity while children over 3 years of age tend to cooperate after explanation of the procedure through play therapy and verbal reassurance. As a result, CT waiting lists have shortened.

Which protocol should be applied?

Planning the examination protocol is essential as this will provide the optimal imaging results that will lead to an accurate diagnosis. Suggested paediatric protocols applied in our institution are displayed in Tables 1, 2, 3, 4 and 5.

\section{Radiation dose}

When dealing with children, the issue of performing a lowdose examination is crucial and of the utmost importance. Radiation dose is a significant issue in paediatrics as it is well established that the life-time cancer mortality risk attributable to CT examinations is considerably higher than in adults [1, 2]. As proposed by the ALARA principle, the selection of appropriate scanning parameters focuses on the optimization of the image quality whilst delivering the lowest possible radiation dose and shifting the risk-benefit balance towards benefit $[1,2,5]$.

Technical parameters that need to be selected for any scan include: thickness of collimation, tube current, and kilovoltage. The thickness of collimation is the minimum
Table 2 Suggested delay times from the injection of contrast medium

\begin{tabular}{lll}
\hline & Manual injection & Pressure injector \\
\hline $\begin{array}{l}\text { Scan initiation } \\
\text { time delay }\end{array}$ & $10 \mathrm{~s}$ from termination & $\begin{array}{c}25 \mathrm{~s} \text { from start } \\
\text { of injection }\end{array}$ \\
Flow rate & & $2 \mathrm{ml} / \mathrm{s}$ \\
Age range & All age groups & All age groups \\
\hline
\end{tabular}

section thickness that can be acquired once the scan is finished and in a 16-row MDCT scanner is usually $1.5 \mathrm{~mm}$. Thinner collimation $(0.75 \mathrm{~mm})$ increases the radiation dose by approximately $30 \%$ with our in-house reduced protocol and is applied only in selected cases of vascular abnormalities, visualization of small structures and in cardiac CT (Tables 1 and 6). The axial images are reconstructed at 5-mm thickness and archived to the PACS system within our hospital. In recent years we have made efforts to standardize low-dose protocols for the children scanned in our institution, and the currently applied parameters are summarized in the Tables 1, 3, 4 and 5. Methods adopted to minimize radiation dose in MDCT include:

1. Applying a dose modulation function, where the system samples the patient thickness and adjusts (i.e. reduces) the exposure accordingly when the tube is in the AP/PA position, as patients are narrower in the frontal than in the side-to-side orientation.

2. Reduction of the kilovoltage to $100 \mathrm{kVp}$ when imaging the thorax. Further reduction to $80 \mathrm{kVp}$ is possible for CTA, but as resolution of the parenchyma is not ideal this is applied only if lung pathology is unlikely.

Table 1 Imaging protocol for the paediatric chest using a 16-row MDCT scanner

\begin{tabular}{lll}
\hline & Routine scan & Combiscan/CTA \\
\hline Indication & Stricture & Cardiovascular anomalies \\
& $\begin{array}{l}\text { Tumour } \\
\text { Tracheomalacia }\end{array}$ & Small tracheobronchial stenoses \\
& Peripheral airways disease & \\
Anatomical area & Thoracic inlet to diaphragm & 0.75 \\
Tube collimation (mm) & 1.5 & 3 \\
Slice width - reconstructed $(\mathrm{mm})$ & 5 & 12 \\
Table feed (mm/rotation) & 24 & $100 \mathrm{kVp}$ \\
Exposure factors & $100 \mathrm{kVp}$ & $20-75$ effective mAs (dependent on patient weight) \\
& $20-75$ effective mAs (dependent on patient weight) & $0.5 \mathrm{~s}$ scan time \\
Respiration & $0.5 \mathrm{~s}$ scan time & Suspended inspiration; single breath-hold where possible \\
Contrast medium & Three to five expiratory scans for tracheomalacia/small airways disease & Yes \\
Algorithm & None & Triggering for CTA \\
& & Soft tissue plus reconstruction on bony algorithm \\
& Soft tissue & for high-resolution CT of the lungs
\end{tabular}


Table 3 Volumetric CT chest scanning parameters according to child's weight when routine and Combiscan protocols are performed

\begin{tabular}{|c|c|c|c|c|c|c|c|c|c|c|}
\hline & \multicolumn{2}{|l|}{$<15 \mathrm{~kg}$} & \multicolumn{2}{|c|}{$15-24 \mathrm{~kg}$} & \multicolumn{2}{|c|}{$25-34 \mathrm{~kg}$} & \multicolumn{2}{|c|}{$35-44 \mathrm{~kg}$} & \multicolumn{2}{|c|}{$45-55 \mathrm{~kg}$} \\
\hline & Volume & Combi & Volume & Combi & Volume & Combi & Volume & Combi & Volume & Combi \\
\hline $\mathrm{kVp}$ & 100 & 100 & 100 & 100 & 100 & 100 & 100 & 100 & 100 & 100 \\
\hline Effective mAs & 20 & 20 & 25 & 25 & 35 & 35 & 55 & 55 & 75 & 75 \\
\hline Collimation (mm) & 1.5 & 0.75 & 1.5 & 0.75 & 1.5 & 0.75 & 1.5 & 0.75 & 1.5 & 0.75 \\
\hline Scan slice width (mm) & 5 & 5 & 5 & 5 & 5 & 5 & 5 & 5 & 8 & 8 \\
\hline Table feed (mm) & 24 & 12 & 24 & 12 & 24 & 12 & 24 & 12 & 24 & 12 \\
\hline Scan time (s) & 0.5 & 0.5 & 0.5 & 0.5 & 0.5 & 0.5 & 0.5 & 0.5 & 0.5 & 0.5 \\
\hline Calculated Effective Dose (mSv)-CT EXPO & 0.9 & 1.0 & 1.13 & 1.31 & 1.58 & 1.75 & 2.48 & 2.75 & 3.38 & 3.75 \\
\hline
\end{tabular}

3. Selecting tube collimation of $1.5 \mathrm{~mm}$. The $0.75-\mathrm{mm}$ collimation improves spatial resolution but, as already mentioned, increases the radiation dose, and is therefore reserved for CTA or where thin-slice reconstruction is indicated.

4. Selecting appropriate mAs selection dependent on the patient's weight or cross-sectional diameter.

Unlike the single-slice scanner, an increase or decrease in table feed time on the MDCT scanner only affects the overall scanning time. An increase in table speed results in a concomitant increase in $\mathrm{mA}$ and this has no effect on the dose delivered. The tube current is automatically compensated to ensure that the preset effective and total $\mathrm{mAs}$ is delivered, i.e. a fast table movement results in an automatic increase in the $\mathrm{mA}$ keeping the mAs constant.

\section{Anatomical coverage}

For imaging of the paediatric thorax, regular coverage extends from the thoracic inlet to the diaphragm. Greater coverage may be warranted in certain clinical cases, such as an extralobar pulmonary sequestration that may derive its blood supply from the upper abdomen.

In order to increase spatial resolution, the field of view (FOV) should closely approximate the cross-sectional area of the body part being studied. A large FOV would result in

Table 4 High-resolution CT chest scanning parameters according to child's weight

\begin{tabular}{llll}
\hline & $<15 \mathrm{~kg}$ & $15-30 \mathrm{~kg}$ & $>30 \mathrm{~kg}$ \\
\hline $\mathrm{kVp}$ & 100 & 100 & 100 \\
Effective mAs & 20 & 30 & 55 \\
Collimation (mm) & 1 & 1 & 1 \\
Scan slice width (mm) & 1 & 1 & 1 \\
Table feed (mm) & 10 & 10 & 10 \\
Scan time (s) & 0.36 & 0.75 & 0.75 \\
Calculated Effective Dose & 0.21 & 0.32 & 0.59 \\
(mSv)-CT EXPO & & & \\
\hline
\end{tabular}

waste of matrix space and partial volume averaging would generate poor quality images [2].

Breathing during scan

Ideally, scans should be performed during suspended inspiration at total lung capacity, but this is not often possible in younger children. Experience has proved quiet breathing to be more successful than the confusion created over attempts to acquire the scans during suspended inspiration [6]. When subtle air-trapping due to small airways disease is suspected, a few supplementary expiratory sections may be obtained. If the child is too young to breathhold, decubitus scans may replace expiratory scans, the dependent lung behaving as the "expiratory" lung and the nondependent lung behaving as the "inspiratory" lung [6]. The acquisition of prone scans is rarely required in older children who may demonstrate hypoventilation changes in the dependent areas of the lungs. General anaesthesia is therefore applied only in a limited number of cases.

\section{Contrast medium}

When imaging the airways, intravenous nonionic contrast material is only administered if paratracheal abnormalities, such as vascular rings, anomalous origin of the pulmonary artery, and mediastinal masses are suspected [2]. "Combiscans" provide the potential to image equally well the lung parenchyma by using the high-resolution techniques (HR) for reconstruction algorithms and the mediastinal structures by injecting contrast medium. However, since $0.75-\mathrm{mm}$ fine collimation is required, there is an inevitable increase in the radiation dose (Table 6) and its application is limited to cases where lung parenchymal detail is of high importance.

An intravenous cannula should preferably be placed in situ on the ward so that the child will not associate CT with venepuncture, especially in those in whom subsequent scans may be required. It is ideally inserted via a peripheral vein of the upper or lower limb. Central lines are used for injection in 
Table 5 CT angiography scanning parameters according to child's weight

\begin{tabular}{llllll}
\hline & $<15 \mathrm{~kg}$ & $15-24 \mathrm{~kg}$ & $25-34 \mathrm{~kg}$ & $35-44 \mathrm{~kg}$ & $45-55 \mathrm{~kg}$ \\
\hline $\mathrm{kVp}$ & 100 & 100 & 100 & 100 & 100 \\
Effective mAs & 20 & 25 & 35 & 40 & 50 \\
Collimation (mm) & 0.75 & 0.75 & 0.75 & 0.75 & 1 \\
Scan slice width (mm) & 1 & 1 & 1 & 15 \\
Table feed (mm) & 15 & 15 & 15 & 0.75 \\
Scan time (s) & 0.75 & 0.75 & 1.75 & 15 \\
Calculated Effective Dose (mSv)-CT EXPO & 1.0 & 1.26 & 2.0 & 0.75 \\
\hline
\end{tabular}

conjunction with clinical agreement and are more desirable as the experience is less unpleasant for the child than having to undergo repeated cannulation. Nonionic contrast medium is administered at a dose of $2.0 \mathrm{ml} / \mathrm{kg}$ up to a maximum of $50 \mathrm{ml}$ via a $20 / 22 \mathrm{G}$ cannula using either a power injector or hand injection (when the cannula is placed in the hand or the wrist). The power injector has the advantage of instilling contrast medium at a constant delivery rate. A flow-rate of $2.0 \mathrm{ml} / \mathrm{s}$ is used rising to $2.5 \mathrm{ml} / \mathrm{s}$ for cardiac studies. The delay time between the start of injection and the start of the scan can be empirical and bolus tracking is routinely used in cardiac CT studies (Table 2).

In children with complex vascular anatomy, the radiologist should be present for the examination. In those children, low-dose planning scans are performed at a predetermined anatomical level and the injection of contrast medium is triggered by the radiologist. The volumetric scan begins when the contrast medium has reached the relevant preselected vascular structure.

\section{Postprocessing}

There are four reconstruction displays available for postprocessing of the volumetric data, which are applied accordingly: multiplanar reformation or reconstruction (MPR), 3-D shaded-surface display (SSD), multiprojective volume reconstruction (MPVR) and 3-D volume rendering (VR). In fact, the axial images include all the information about the anatomy of the airways that is provided with 2-D and 3-D reformats. However, postprocessing gives added value to imaging since the axial scans that need to be studied are usually numerous, and oblique structures as well as interfaces and surfaces parallel to the axial plane are poorly demonstrated and sometimes occult.

\section{Multiplanar reformations}

These provide additional diagnostic information in different planes and are as accurate as the axial scans due to the nature of the isovolumetric acquisition of the data [3]. MPRs are one-voxel-thick 2-D tomographic sections that can be displayed in coronal, sagittal, or parasagittal planes or in a single tomographic "curved" plane, along the axis of a structure of interest, e.g. a bronchus or a feeding vessel (Figs. 1, 2, 3, 4, 5, 6, 7, 8, 9 and 10) [2, 3]. They are realtime easy-to-reconstruct images, producible as soon as the axial sections are completed. They generally improve our perception of images and give information that although contained in transverse images, is sometimes less effectively displayed. Their diagnostic value is substantial in demonstrating and documenting the presence of small focal lesions, defining the vertical extent of a bronchial stenosis, which may go undiagnosed from the axial source CT images (Figs. 4, 5 and 6), and are invaluable prior to surgical remodelling of vascular rings and the tracheobronchial tree [3]. However, to avoid misinterpretations due to partial volume effect, e.g. overestimation of the degree of a stenosis, overlapping and thinner cuts should be applied when processing the raw data. Likewise, when processing curved MPRs, the trace should be centred within the lumen of interest to avoid anatomical distortion.

Table 6 Dose comparison for different scanning protocols in a phantom study in our institution

\begin{tabular}{|c|c|c|c|c|c|c|c|c|c|c|}
\hline \multirow[t]{3}{*}{ Weight range $(\mathrm{kg})$} & \multicolumn{10}{|c|}{ Effective dose (mSv) } \\
\hline & \multicolumn{2}{|c|}{ Volume $(1.5 \mathrm{~mm})$} & \multicolumn{2}{|c|}{ Combi $(0.75 \mathrm{~mm})$} & \multicolumn{2}{|c|}{ High-resolution CT } & \multicolumn{2}{|l|}{ CTA } & \multicolumn{2}{|c|}{ Chest radiography } \\
\hline & Male & Female & Male & Female & Male & Female & Male & Female & AP & Lateral \\
\hline$<15$ & 0.77 & 0.90 & 0.9 & 1.05 & 0.36 & 0.42 & 1.30 & 1.51 & 0.00487 & 0.00799 \\
\hline $15-24$ & 0.93 & 1.09 & 1.13 & 1.31 & 0.36 & 0.42 & 1.62 & 1.89 & 0.00874 & 0.01086 \\
\hline $25-34$ & 1.34 & 1.56 & 1.58 & 1.84 & 0.54 & 0.63 & 2.24 & 2.62 & 0.01163 & 0.00968 \\
\hline $35-44$ & 2.11 & 2.46 & 2.48 & 2.89 & 1.00 & 1.17 & 2.57 & 3.0 & 0.01769 & 0.01452 \\
\hline
\end{tabular}




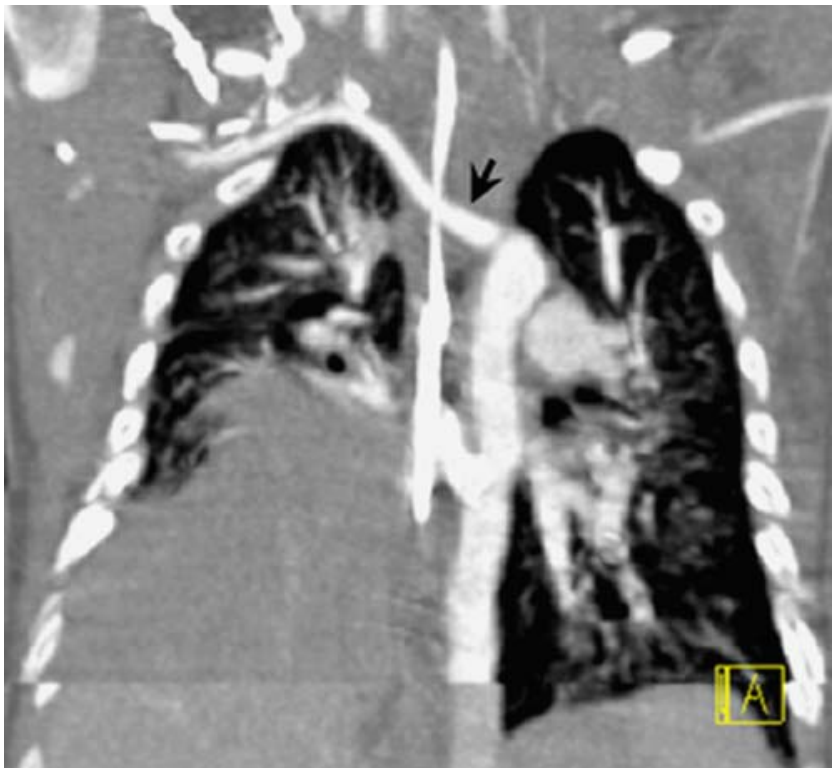

Fig. 1 Coronal oblique MPR in 4.5-month-old boy with trisomy 21 . There is right lung hypoplasia and diaphragmatic eventration. An incidental finding is an aberrant right subclavian artery (arrow)

\section{3-D imaging}

This is a diagnostic tool only in certain cases as it usually requires more time and postprocessing skills to provide information already included and demonstrated in the axial images and the MPRs. There is no doubt, however, that the 3-D reformatted images may further increase the diagnostic confidence which eventually affects patient management, particularly pertinent in presurgical assessment. Communi-

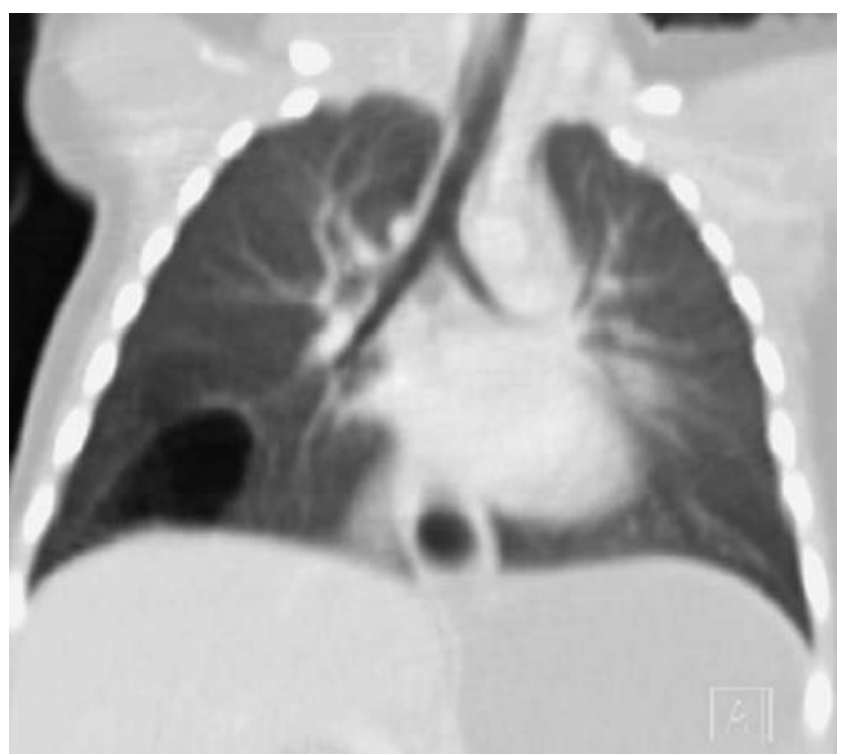

Fig. 2 Coronal MPR of the chest in a child with a history of recurrent infections due to a congenital pulmonary airway malformation. A thin-walled multicystic lesion in the right lower lobe is shown cation with the referring clinicians is simplified as the images portray the spatial relationships of important anatomical structures.

\section{3-D shaded-surface display techniques}

These are only applied in the imaging of the central airways and they are usually more visually impressive than clinically useful. Their generation from original data is time-consuming and they carry the risk of loss of density information due to problems with thresholding.

\section{Multiplanar volume reconstructions}

These are "selective" 3-D images that resemble the 2-D MPRs and depict peripheral airways better than individual sections. With this technique, a combination of the spatial resolution of thin sections and the anatomical display of thicker slices is achieved, and all the information acquired in the raw data set is used. The routine CT images are combined in multiples to create an image thicker in voxels, the volume "slab", which constitutes an interactive sum of axial, coronal and sagittal reconstructed sections [2, 3]. By using different algorithms and setting thresholds, maximum or minimum intensity voxels can be highlighted within the slab. For the evaluation of the airways, minimum intensity projections within the slab are usually applied (Fig. 11) [2]. Slabs are useful in detecting and localizing micronodular or microtubular patterns and in analyzing mild forms of uneven attenuation of the lungs [7]. However, they are time-consuming and should be reserved for cases of complex morphology, to give the clinicians a comprehensive multilevel roadmap 3-D image.

\section{3-D volume rendering}

These represent the main technique for 3-D reformatting of the airways and the vascular structures of the mediastinum. The VR technique is particularly useful for displaying structures that course parallel or oblique to the transverse plane and those that develop or extend into multiple planes $[2,3]$. However, preliminary editing is still necessary and can be time consuming, altering work patterns in CT. In VR, different anatomical tissues are represented by proportional values that are assigned to every voxel and depend on the range of tissue attenuation values in the original data set. Voxels are selected by the probability of belonging to the object of interest; thus they are displayed in different colours or several shades of grey, different transparency or opacity. Transition from the reconstructed surface to the surrounding media is gradual and the depiction of interfaces, like the inner tracheal lining, is supposed to approach their true appearances. Although this 3-D segmentation 

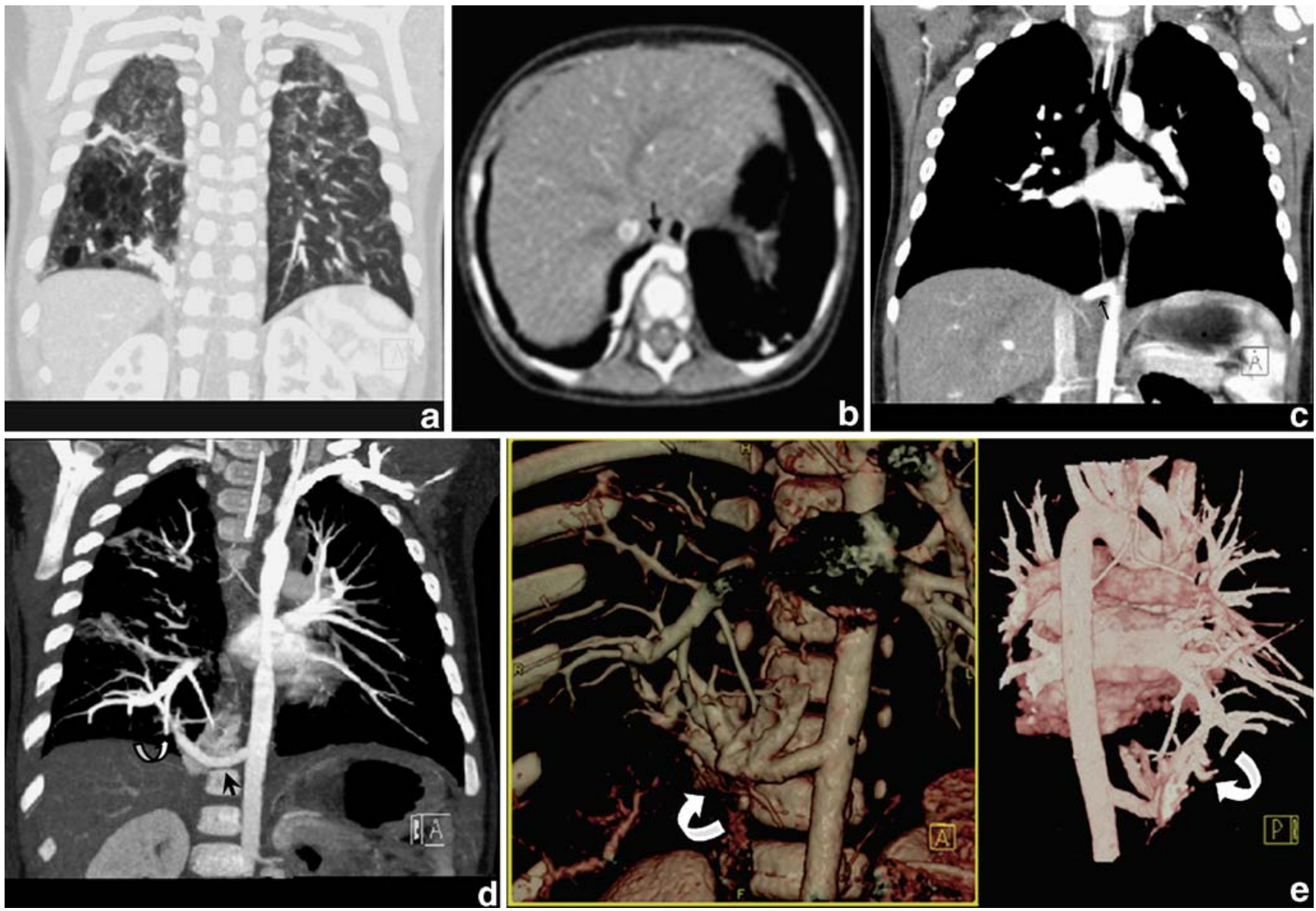

Fig. 3 Combiscan in an 11-month-old child with a congenital pulmonary airway malformation in the right lower lobe. a Coronal MPR in lung window setting shows the air-filled cystic component of the lesion. b, c Axial CT slice (b) and coronal MPR (c) showing a large arterial feeder (arrow). This is, however, visualized and

appreciated better with an oblique MIP along the axis of the vessel (d) and with coronal anterior and posterior 3-D VR (e). These images additionally demonstrate the pulmonary venous drainage of the lesion, i.e. intralobar sequestration (curved arrows)

technique is better and more complicated than the others previously mentioned, some information is lost during processing, so that the axial images are still indispensable for the radiologist to assess extraluminal disease and identify artefacts. Three-dimensional VR-reconstructed images are attractive and appealing to the clinicians as they may better illustrate short focal areas of narrowing, the craniocaudal length of a tracheobronchial stenosis, and complex congenital cardiovascular and tracheobronchial anomalies (Figs. 2, 3, 6, 7, 11, 12).

\section{Virtual bronchoscopy}

The inner surface of the air-containing tracheobronchial tree can be displayed with virtual bronchoscopy (VB), which is performed with either polygonal SSD or direct VR. VB is a noninvasive and accurate technique that can provide "bronchoscopic" views of the central and the peripheral airways (Figs. 4 and 13). This technique, like fibreoptic bronchoscopy, is considered supplementary to CT and referral to the axial sections is again valuable in recognizing artefacts and in gaining perception of orientation within the virtual airways. VB uses perspective surface rendering, which takes advantage of the natural contrast between the airway and the surrounding tissues [1]. The point for viewing is located intraluminally so that external structures do not overlap and editing takes shorter time periods. Sub-millimetre $(0.75 \mathrm{~mm})$ slice thickness allows deeper penetration and visualization of the bronchial surface down to bronchial diameters of less than $5 \mathrm{~mm}$; it is, however, associated with an inevitable increase in radiation dose [4].

Again, VB practically never really adds anything new to the established diagnosis. However, pulmonologists may prefer and relate to these images in conjunction with the axial or the reformatted ones [2]. In practice, VB is of limited value, reserved for cases where bronchoscopy is not applicable (children in whom traditional bronchoscopy 
Fig. 4 Congenital tracheal stenosis in a 3-year-old girl with trisomy 21. a Series of axial slices demonstrating stenosis in the central third of the trachea. b This is more sensitively demonstrated on coronal MPR, which correlates well with the bronchographic appearances (c) (arrows). d Virtual bronchoscopy also demonstrates mild stenosis of the supracarinal portion of the trachea (arrowheads)
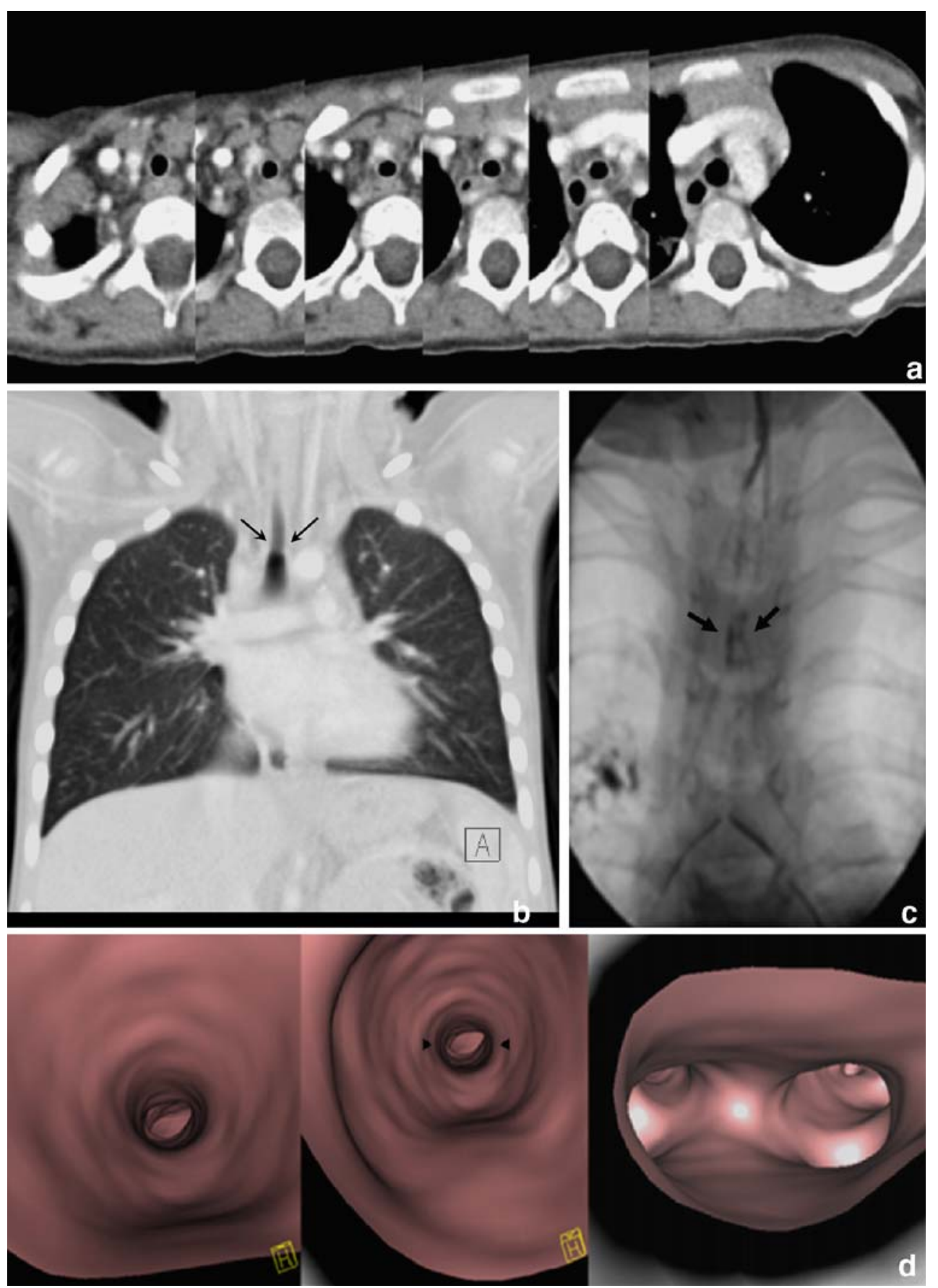

presents a risk) or precise navigation is not possible to guide airway interventional procedures, and in emergencies such as infant airway (tracheal) stenosis which cannot be otherwise evaluated [4]. The images produced resemble the actual bronchoscopic ones and can additionally "advance" distal to an airway obstruction, where the real endoscope cannot penetrate $[3,8]$. However, when compared to fibreoptic bronchoscopy, VB does not allow any therapeutic manoeuvring, is incapable of detecting endoluminal lesions smaller than 2-3 $\mathrm{mm}$ that may go undetected due to the partial volume effect, and provides limited information about mucosal detail (morphology, vascularity and colour), making differentiation between pathological processes and retained secretions difficult $[1-4,9,10]$. There is consensus that measurements should be performed only on 2-D sections as they may otherwise be inaccurate $[3,8]$. The technique is additionally affected by the partial volume effect, which may lead to misinterpretation of severe stenoses as occlusions, and the threshold level is therefore of importance for displaying accurate simulations [8]. 
Fig. 5 Chest CT $(1.5 \mathrm{~mm}$ collimation following intravenous injection of contrast medium) in 10-month-old boy with recurrent wheezing due to congenital tracheal stenosis treated previously with tracheoplasty. There is mild stenosis of the trachea and the origin of the right main bronchus, which is appreciated with difficulty on the axial scans(a), but is nicely shown in coronal MPR (arrow) (b). Virtual bronchoscopy in this case was unnecessary. Bronchography (c) and bronchoscopy confirmed stenosis in the right main bronchus (arrow) that was caused by granulation tissue
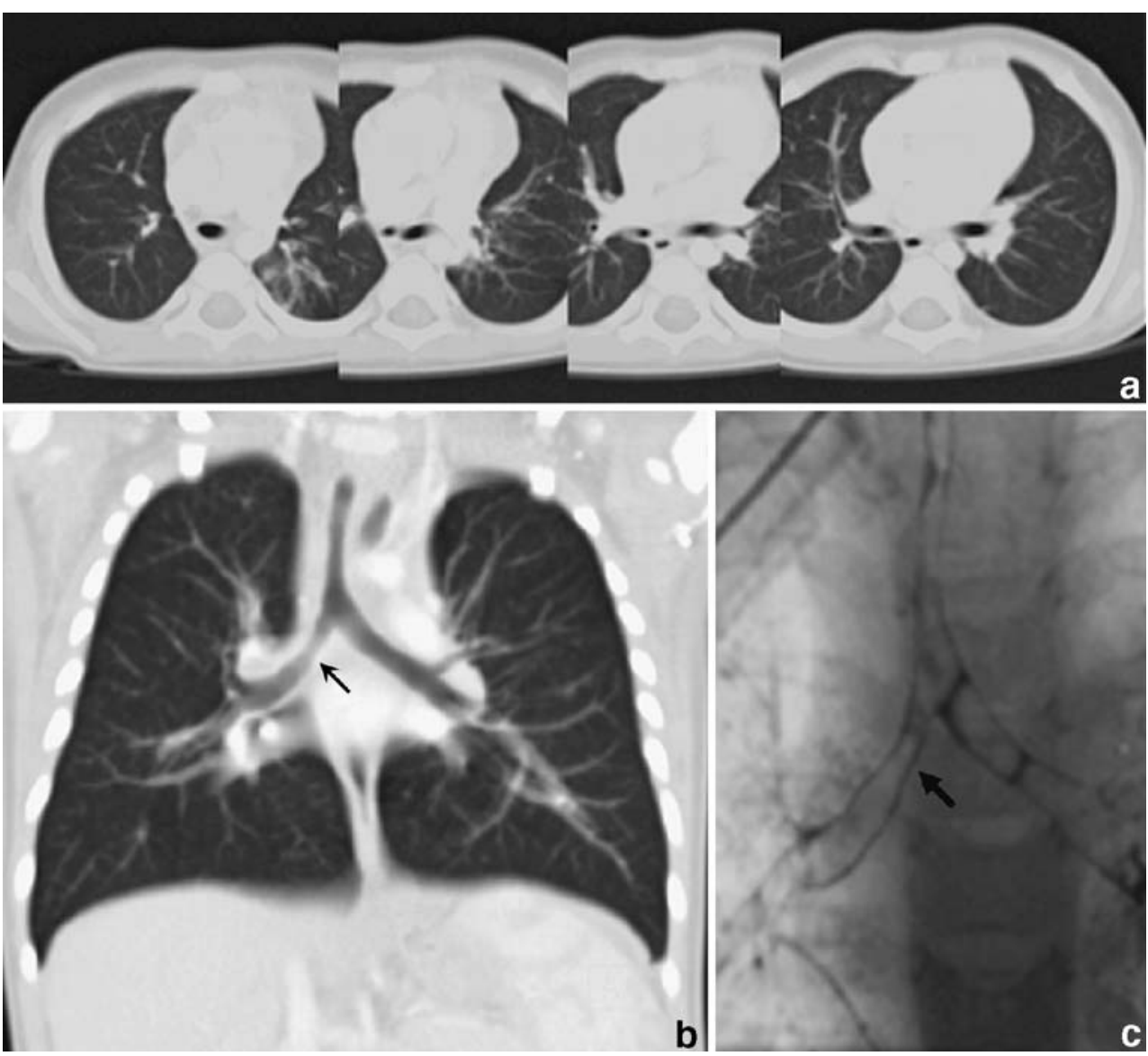

\section{Dynamic and functional inspiratory and expiratory scanning}

In conjunction with single-breath-hold MDCT, dynamic and functional inspiratory and expiratory scanning helps identify strictures, areas of air-trapping and tracheobronchomalacia, but is rarely applied in paediatric practice because of the associated radiation burden [2].

\section{Peripheral airways}

Modern volumetric techniques have partially overcome the difficulty in demonstrating 3-D images of the peripheral airways. Data are acquired and reconstructed at a later time, allowing thin-slice high-resolution images at any level, so that a whole affected bronchiectatic segment can be displayed on a single MPR/VR oblique section (Fig. 10) with images that resemble conventional bronchography and may influence the approach for tissue sampling [3, 7]. If there is suspicion of small-airways disease, some additional expiratory slices may be useful. Whatever the application we perform, all the techniques are considered as an adjunct to conventional HRCT evaluation of the peripheral airways that allows accurate and precise assessment of diffuse lung disease at very low doses equivalent to approximately ten chest radiographs $[3,6]$.

\section{Clinical applications of volumetric imaging of the airways in children}

Selection of the most appropriate CT protocol for each individual case is paramount in imaging of the airways in children. Ultimately, the best protocol is the one that provides the most relevant information at the lowest radiation burden possible. The indications include: (1) congenital bronchial anomalies (e.g. accessory bronchi, bronchial hypoplasia and atresia, and bronchopulmonary foregut malformations), (2) tracheomalacia, (3) tracheobronchial strictures (congenital and acquired) or tumours, and (4) peripheral (small) airways disease.

Tracheobronchial anomalies

Approximately $1-12 \%$ of adult patients who undergo bronchoscopy demonstrate some form of congenital variant $[1,11]$. Tracheobronchial anomalies may be associated with 
Fig. 6 Cardiac CT $(0.75$ collimation after intravenous injection of contrast medium) in a 6-month-old girl with absent pulmonary valve syndrome. a Axial contrast-enhanced CT shows massive dilatation of the main and the left pulmonary arteries with possible compression of the left main bronchus. This is also appreciated on serial oblique MIPs (b), but becomes more apparent on the oblique MIP along the axis of the left main bronchus (c) (black arrow) and on the 3-D VR (d)

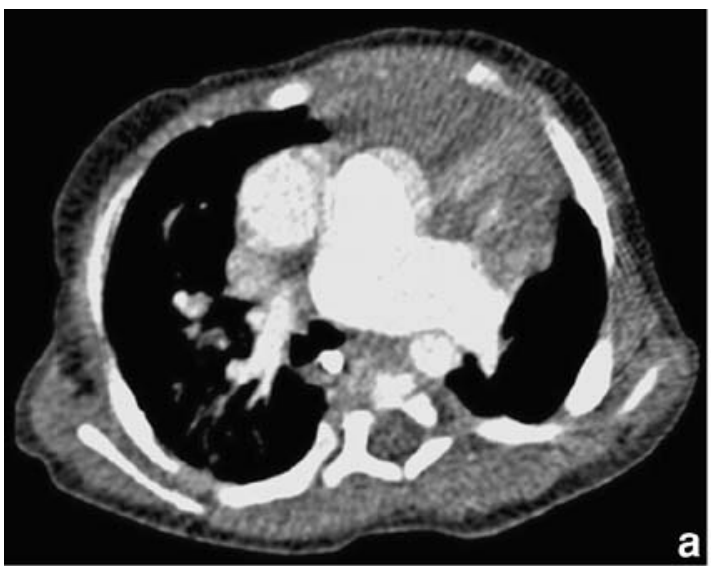
(white arrow)
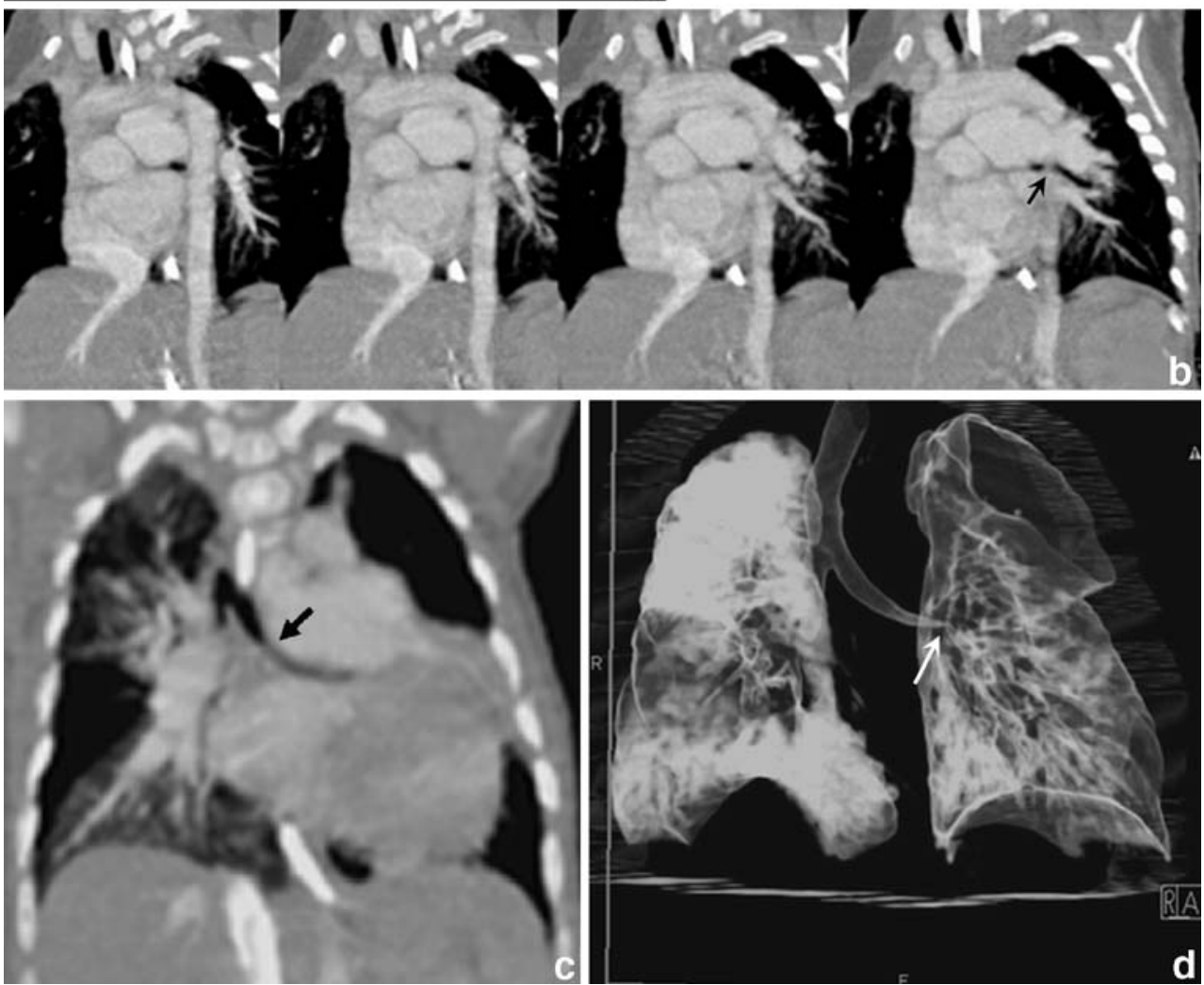

recurrent episodes of pulmonary infection and airway obstruction and usually go undiagnosed with conventional imaging $[1,12]$.

\section{Tracheal bronchus}

Tracheal bronchus, with an incidence of $0.1-5 \%$, represents an aberrant bronchus that usually arises from the right tracheal wall above the carina and ventilates the apical segment of the upper lobe (Fig. 13) $[1,13]$. If the right upper-lobe bronchus has a normal trifurcation then the tracheal bronchus is supernumerary and may end blindly; it is also called a tracheal diverticulum. Left tracheal bronchus has also been reported [13]. Its presence may be associated in some patients with other abnormalities of the lung or within the spectrum of the VACTREL ( $V$ vertebral, $A$ anorectal atresia, $C$ cardiac, $T$ tracheobronchial, $R$ renal, $E$ esophageal, $L$ lumbosacral/limb abnormalities) association. Diagnosis of tracheal bronchus should be considered early in the clinical course of intubated patients with recurrent right upper-lobe complications [11]. Coronal MPRs are particularly helpful in demonstrating the presence of a tracheal bronchus, which may go undetected on axial images. Findings can be additionally supported with VB. 
Fig. 7 Combiscan of the chest in 15-year-old girl with laryngeal/pulmonary papillomatosis. a There is irregular thickening and narrowing of the proximal tracheal wall that is well demonstrated on axial imaging. b, c Coronal MPRs additionally demonstrate intrapulmonary cavitating lesions. Same findings are impressively demonstrated on 3-D VR (d)
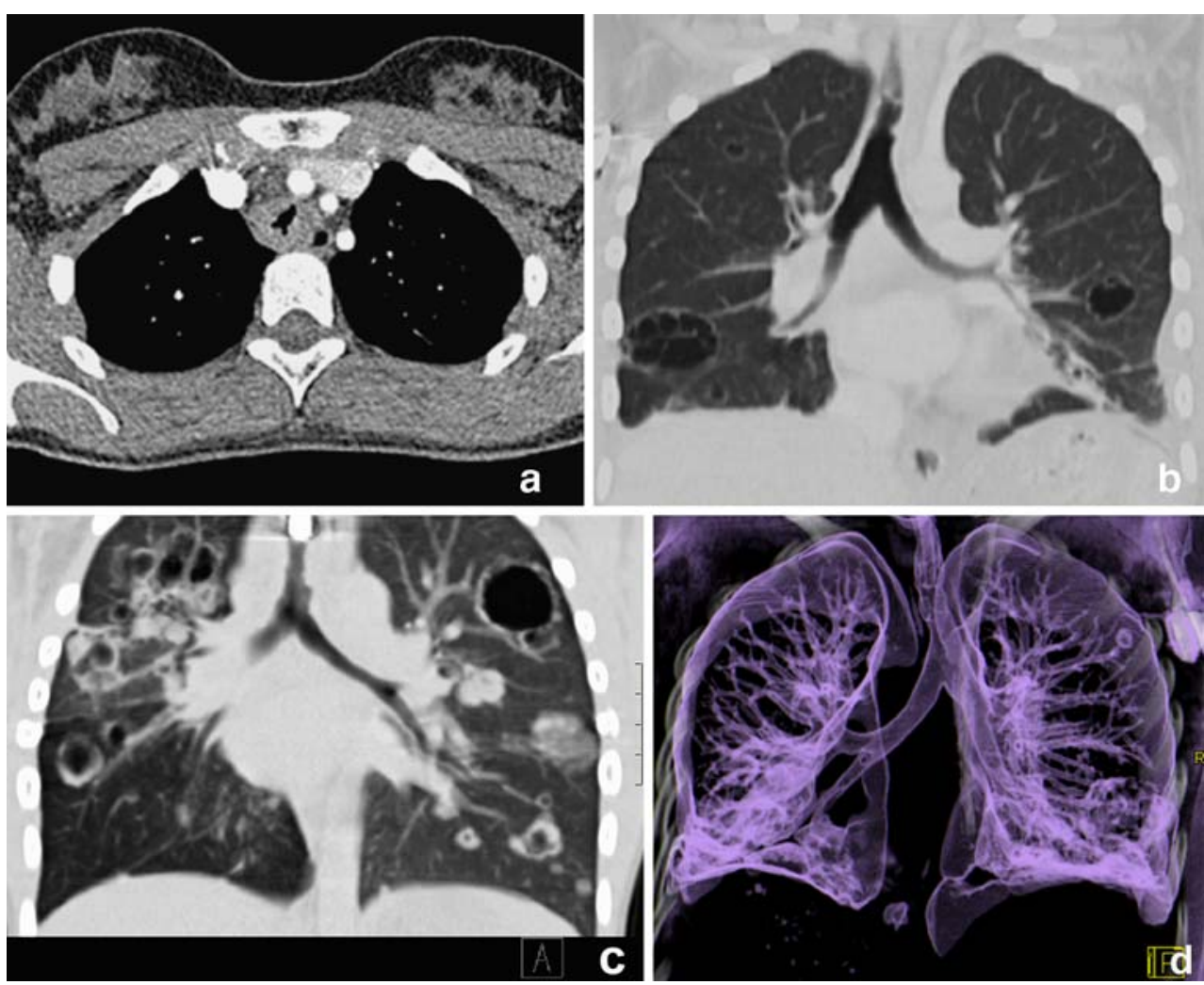

\section{Bronchial atresia}

Bronchial atresia may present with a variety of findings depending on the child's age (water-density mass in newborns, bronchocele and air-trapping during childhood, and solitary pulmonary nodule or area of emphysema in adults). In doubtful cases, MPRs help identify mucoid impaction and bronchocele as a branching structure radiating from the hilum [13].

\section{Bronchopulmonary foregut malformations}

These are anomalies of pulmonary development that are due to abnormal budding of the embryonic foregut and tracheobronchial tree. They include duplication cysts characterized by an isolated portion of lung tissue communicating with the upper gastrointestinal tract or the central nervous system such as bronchogenic cysts, enteric cysts, and neurenteric cysts. Symptoms are usually provoked by the size and location of the cysts, which may cause compression of the trachea or bronchi leading to distal collapse and air trapping. Infection is less commonly encountered.

\section{Pulmonary underdevelopment}

The spectrum of pulmonary underdevelopment includes agenesis of the bronchus and lung (pulmonary agenesis), presence of a rudimentary blind-ending bronchus without lung tissue (pulmonary aplasia) and bronchial hypoplasia
Fig. 8 Myofibroelastic tumour in a child with a history of cough and haemoptysis. Axial imaging (a) and coronal (b) MPR demonstrate infiltration of the right main bronchus from a heterogeneous soft-tissue mass
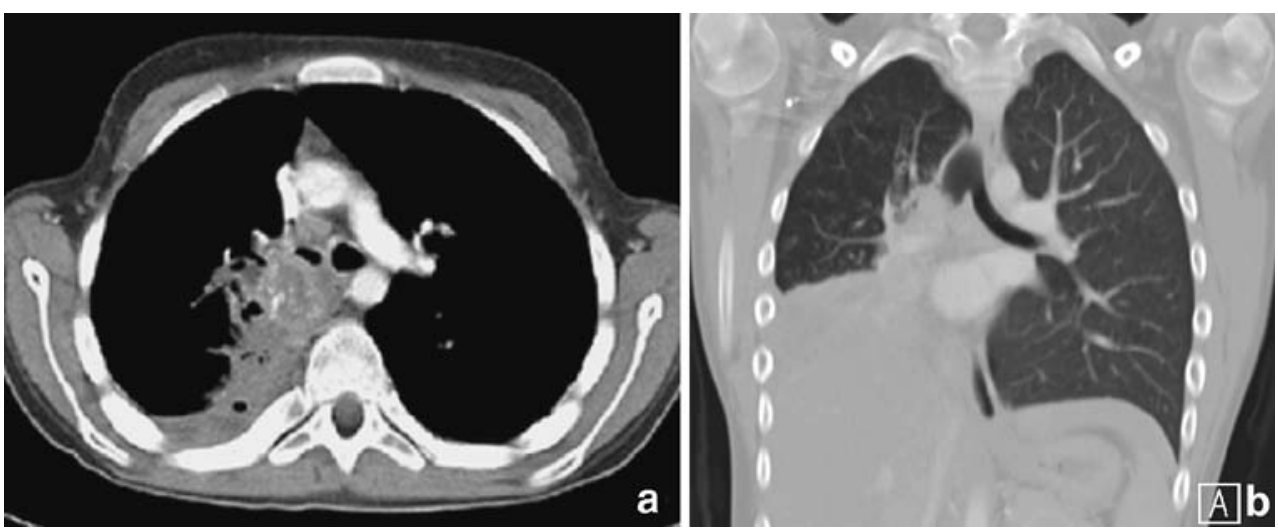


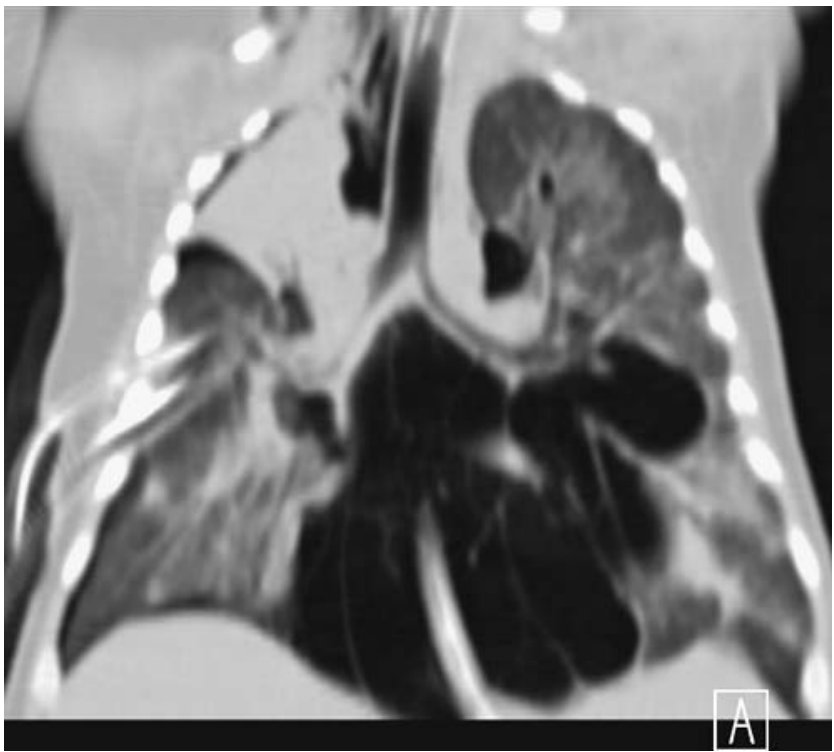

Fig. 9 Coronal MPR shows in detail the extent of the pneumomediastinum with associated surgical emphysema and right pneumothorax in a child with severe combined immune deficiency (SCID) and Pneumocystis carinii pneumonia

with reduction of lung tissue of variable degree (pulmonary hypoplasia) (Fig. 1) [13].

\section{Scimitar syndrome}

The scimitar syndrome, also called venolobar syndrome and hypogenetic lung syndrome, is a rare congenital cardiovascular anomaly involving the right lung. It

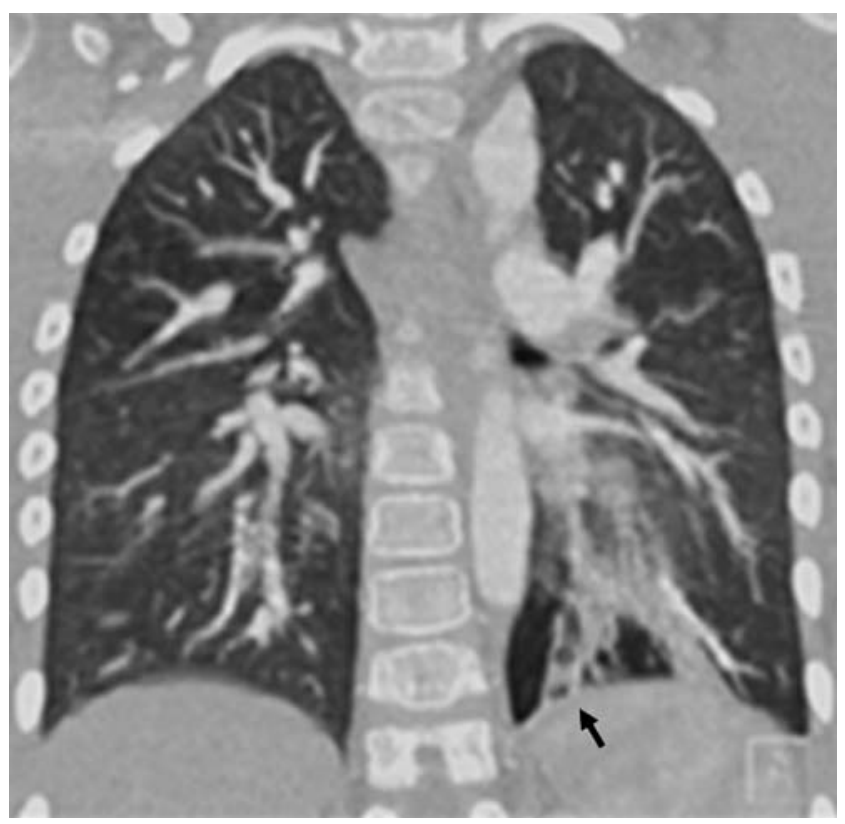

Fig. 10 Coronal MPR in lung window setting demonstrates left lower lobe bronchiectasis (arrow) in a boy with a history of chronic aspiration consists of ipsilateral anomalous pulmonary drainage of part of the right lung into the inferior vena cava, hypoplasia of the right lung, dextrorotation of the heart, hypoplasia of the right pulmonary artery, and anomalous systemic arterial supply to the lower lobe of the right lung from the subdiaphragmatic aorta or its main branches. It may be associated with abnormal bronchial anatomy, abnormal diaphragm, hemivertebrae, and anomalies of the genitourinary tract. CT with MPRs shows the size of the right hemithorax, the associated bronchial anomalies, and the anomalous pulmonary (scimitar) vein [13].

\section{Sequestration spectrum}

The sequestration spectrum includes congenital pulmonary airway malformations (CPAMs), anomalies previously termed congenital cystic adenomatoid malformations, the pulmonary sequestrations and also hybrid lesions that histologically consist of both entities (Figs. 2 and 3). CT defines their location, extent and anatomical relationships, and evaluates their density. MPRs and VRs can impressively demonstrate the arterial feeder from a subphrenic aortic branch and detect the venous drainage into the systemic or pulmonary circulation for the extra- and intralobar sequestrations, respectively (Fig. 3). Enhancement of the cystic wall implies infection.

\section{Tracheomalacia}

Tracheomalacia is a condition that refers to softening of the tracheal wall. This is caused from an abnormality of the cartilaginous ring and hypotonia of the myoelastic elements. There is dynamic collapse of the trachea during expiration which leads to airway obstruction with development of wheeze, cough, stridor, dyspnoea, cyanosis and recurrent respiratory infections. Primary tracheomalacia is thought to be caused by congenital immaturity of the tracheal cartilage and may be associated with other developmental defects, such as a vascular ring/compression or tracheo-oesophageal fistula. The natural history shows that it improves by the end of the first year. In secondary tracheomalacia, previously normal cartilage undergoes degeneration [13].

Tracheobronchial strictures

\section{Congenital tracheal stenosis}

Congenital tracheal stenosis is a rare disorder characterized by a fixed tracheal narrowing caused by complete tracheal cartilage rings (Fig. 4). Approximately $50 \%$ of congenital tracheal stenoses are focal, located usually in the lower 
Fig. 11 Complete vascular ring around the trachea and the oesophagus formed by a double aortic arch. a Diagrammatic representation of the abnormality. b Corresponding contrast-enhanced axial image at the level of the double aortic arch, the right part being the dominant. c 3-D VR image of the double aortic arch from a left anterior oblique view. Volume slab (d) and coronal MPR (e) additionally demonstrate the resulting significant narrowing of the trachea
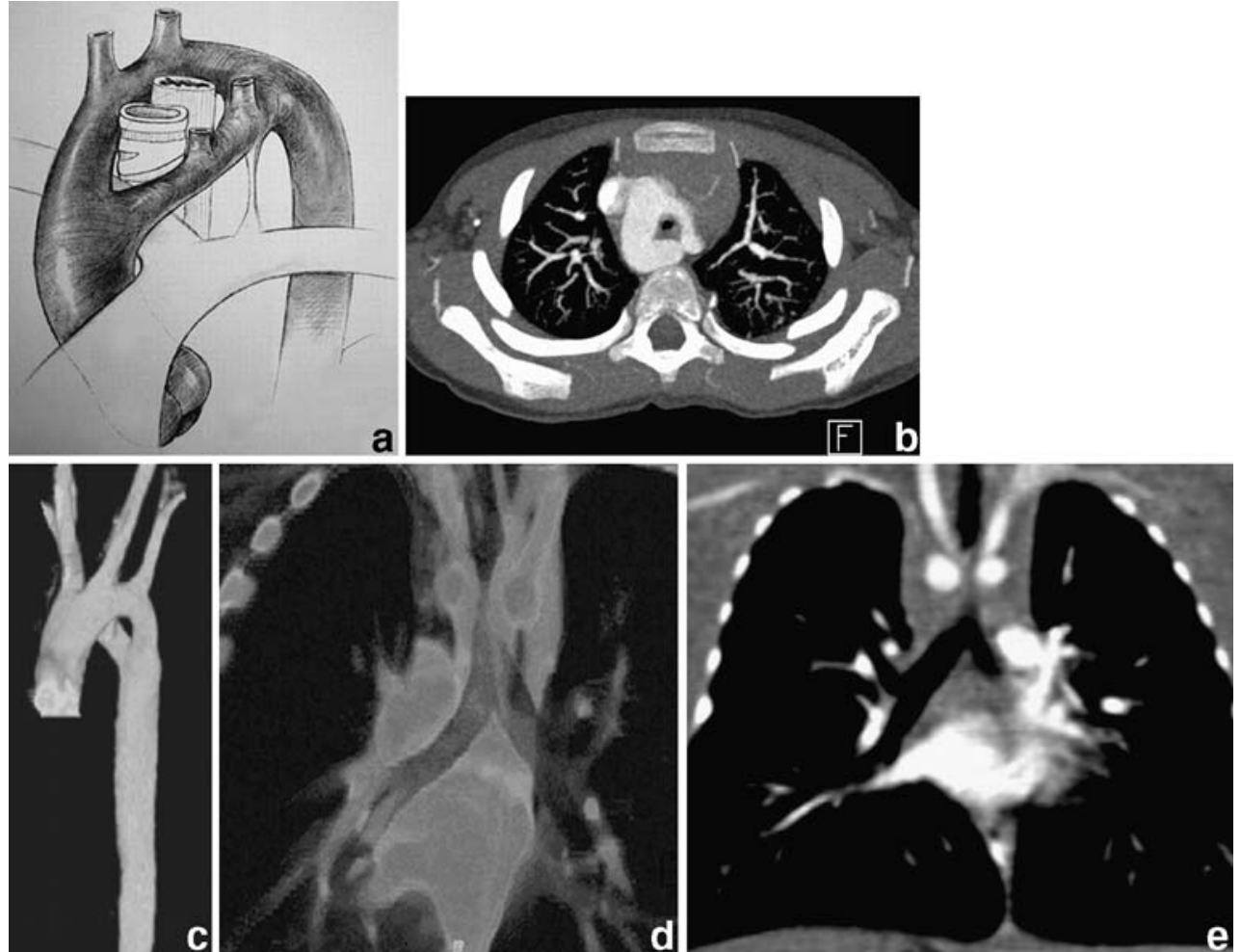

third of the trachea, $30 \%$ are generalized and $20 \%$ are funnel-shaped [13]. The bronchi are usually of normal size. This abnormality which may be associated with other anomalies, more commonly pulmonary artery sling, should be suspected when a distal tracheal stenosis is recognized in an infant [13].

\section{Congenital lobar emphysema/overinflation}

Areas of malacia or stenosis of the bronchial cartilage are thought to be the aetiological factors in the development of congenital lobar emphysema/overinflation through a checkvalve mechanism, which is characterized by progressive distension/hyperinflation of a lobe, most commonly the left upper lobe. CT will typically show hyperinflation of the affected lobe without destruction of the alveolar walls and will help to differentiate this condition from others (Fig. 14).

\section{Acquired tracheobronchial stenoses}

Acquired tracheobronchial stenoses may result from prolonged intubation (post-intubation webs) or at the anastomosis sites following surgical intervention/lung transplantation. In patients in whom the axis of the stenosis is vertical or slightly oblique it is usually difficult to perceive on the axial

Fig. 12 a Contrast enhanced 16-slice MCDT (0.75-mm collimation) showing marked tracheal narrowing and an aberrant origin of the left pulmonary artery from the right pulmonary artery (pulmonary sling). b 3D VR demonstrating an elongated and stenotic trachea with identification of a separate bronchus for the right upper lobe
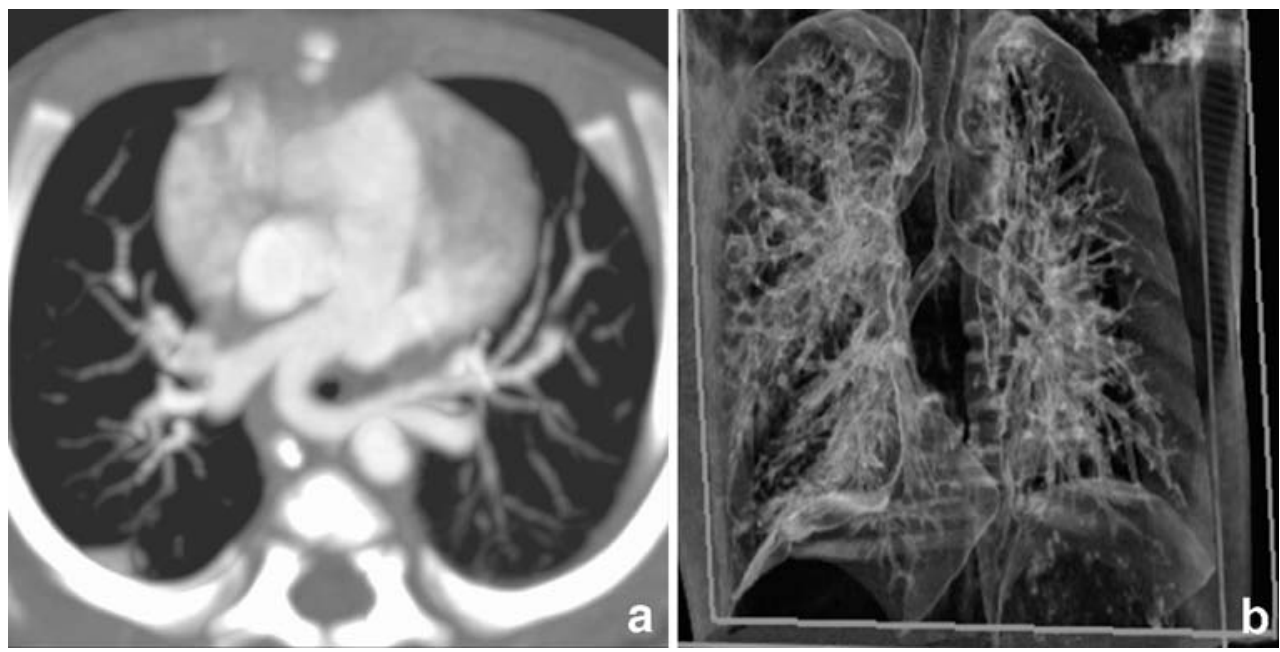
Fig. 13 Accessory bronchus. a Bronchography and $\mathbf{b}$ volume rendered 16-slice VB demonstrating an accessory bronchus (curved arrows). On the MDCT image at the level of the carina, a more cephalad origin of the apical accessory bronchus is noted
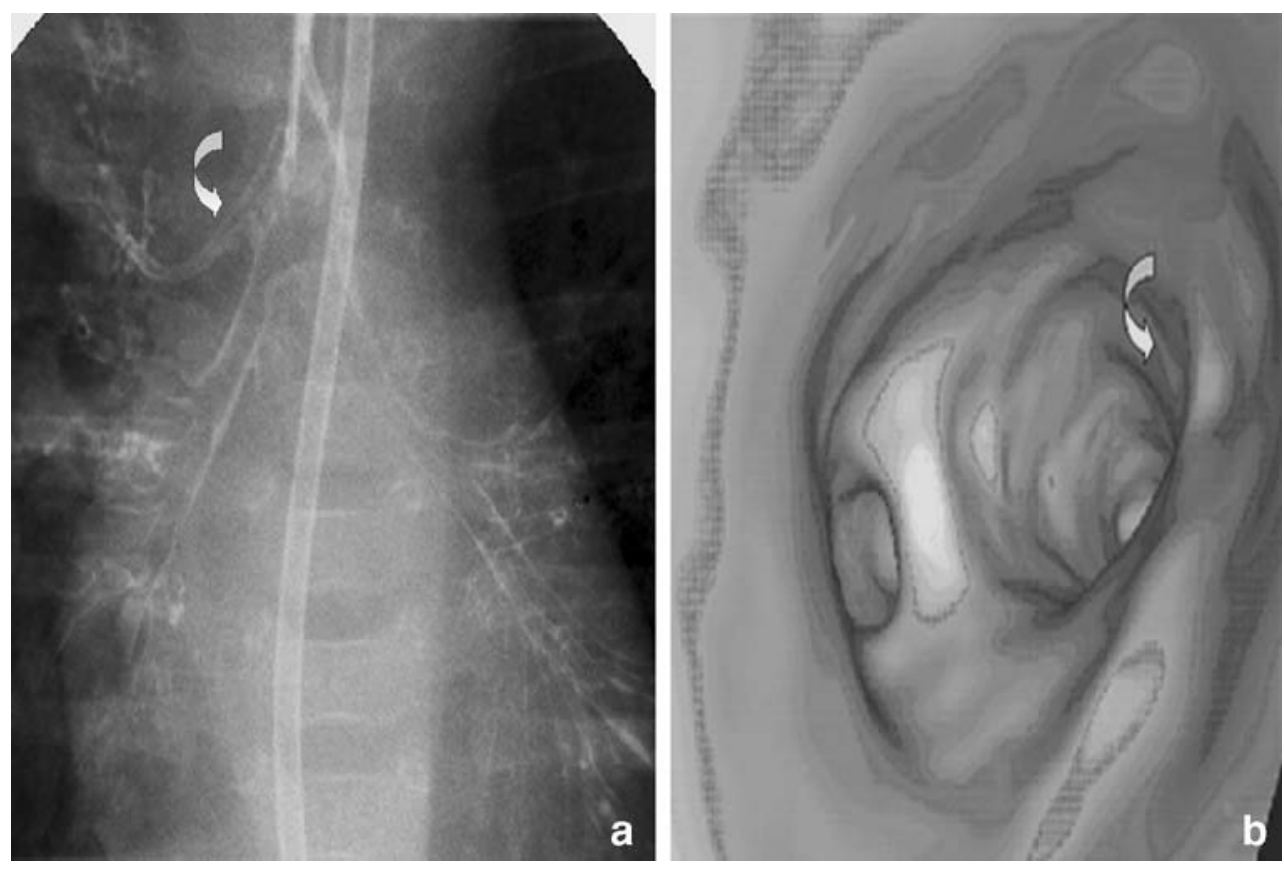

images alone, and MPRs/VRs are useful adjunctive tools (Fig. 5).

\section{Compression of the airways of cardiovascular origin}

This is a relatively common complication of cardiovascular diseases that may go unrecognized for some time. Compression is caused either because of an underlying anomalous anatomical relationship between the tracheobronchial tree and the vascular structures, e.g. a double aortic arch, or is extrinsic, caused by enlarged cardiac or pulmonary

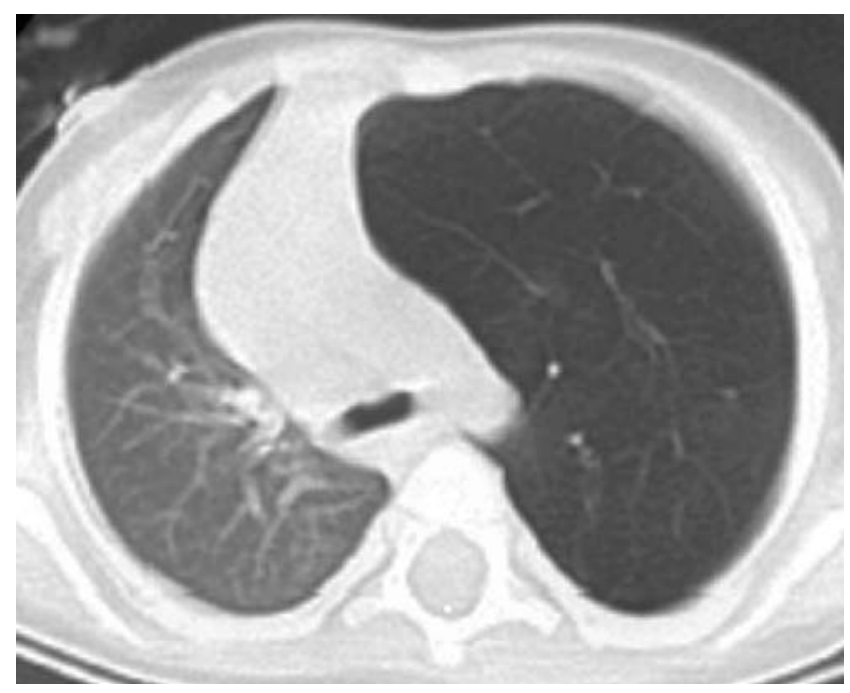

Fig. 14 Axial CT of an infant with mediastinal shift shown on a chest radiograph showing hyperinflation of the left upper lobe without destruction of the alveolar walls, consistent with congenital lobar emphysema vascular structures, e.g. congenital heart disease, dilated pulmonary arteries (Fig. 6). In children with unexplained respiratory distress, stridor, wheezing, dysphagia and apnoea, a high index of suspicion is required to identify these lesions that cause mechanical airway compression $[14,15]$.

A vascular ring is an aortic arch anomaly in which the trachea and the oesophagus are completely surrounded by vascular structures. They are formed because components of the aortic arch complex persist or regress abnormally. If the airway is incompletely encircled then a vascular sling is produced [13-15].

The most common and clinically serious type of vascular ring is the double aortic arch, which represents a persistence of both right and left embryonic fourth arches joining the aortic portion of the truncoaortic sac to their respective dorsal aortae [14]. It typically consists of a right and a left arch that encircle the trachea and oesophagus in a tight ring, joining distally to form a common descending aorta (Fig. 11). Variations of this anomaly have been reported, e.g. hypoplasia of one arch [13].

Another vascular sling can be produced by an abnormal origin of the left pulmonary artery from the right pulmonary artery (Fig. 12). As the former crosses to the left, it encircles and compresses the right main bronchus and the trachea and occasionally the left main bronchus. This condition is usually associated with other congenital anomalies [14].

\section{Foreign body aspiration}

This is one of the most common and potentially life threatening events in children, accounting for $7 \%$ of lethal 
accidents in infants aged 1-3 years [16]. MDCT of the chest for the detection of radiolucent foreign bodies is not the investigation of first choice and not routinely used unless a pulmonary infiltrate that fails to resolve within the normal recovery period of 10-14 days is present [16]. It is vital to remember that actual bronchoscopy should be performed even in cases of low clinical suspicion and no direct history since the risk of overlooking foreign body aspiration may be lethal and may cause long-term pulmonary damage.

\section{Local extension of neoplasms}

MPRs/VRs with coevaluation of the axial images are the method of choice for staging of hilar or mediastinal neoplasms. They demonstrate interfaces accurately, e.g. the relationship of the lesions to the bronchovascular walls and the presence of mediastinal lymph nodes, since they maintain voxel density values (Figs. 7 and 8). More complex rendering techniques are not usually required [3].

\section{Peribronchial air collections}

MPRs/VRs are helpful in demonstrating small extraluminal air collections and leakages of surgical anastomoses, especially in orthotopic lung or heart/lung transplant patients (Fig. 9). They can impressively show fistulous paths that may be indiscernible on axial images and affect patient management [3]. Additionally, by correlating with VB the precise location of the leak can be traced prior to actual bronchoscopy.

\section{Peripheral airways}

MPRs/VRs using MDCT technology have achieved more accurate visualization of the distal airways. The VR technique is applied in thin, overlapping, transverse reconstructed sections using a high-resolution algorithm. This is particularly helpful in the search for bronchiectasis where a single oblique MPR/VR section may include the whole portion of the affected bronchi (Fig. 10). Additionally, when planning the best approach for tissue sampling the relation between peripheral nodules and the afferent bronchus is better appreciated using the MIP/VR sections. Although the applications of conventional HRCT for the evaluation of the peripheral airways are beyond the scope of this review article, the value of HRCT should not be overlooked. When performed individually, HRCT is considerably less of a radiation burden than MDCT (Table 6), but with the standard HRCT protocol, limited slices are performed and the imaging of the mediastinum is insufficient. On the contrary, the Combiscan MDCT protocol with the possibility of HR reconstructions allows detailed parenchymal evaluation of the whole of the chest and simultaneous imaging of the mediastinum; however, there is the disadvantage of extra radiation burden (Table 6).

\section{Conclusion}

MDCT with 2-D and 3-D reconstructed imaging has enhanced the applications of CT in imaging of the chest in children. Obtaining high-quality scans should always be attempted at the lowest radiation dose possible. CT and bronchoscopy are supplementary examinations in the diagnostic work-up of children with tracheobronchial pathology. Although 2-D and 3-D rendering techniques are not the first-line diagnostic tools, they significantly reinforce the confidence in a diagnosis even in complex cases. In specific scenarios, they may supply information that is more easily interpreted among the different specialties than conventional axial scans. In the future, virtual tracheobronchial endoscopy is anticipated to be applied for interactive virtual-reality guidance in surgical procedures of the airways.

\section{References}

1. Heyer CM, Kagel T, Lemburg SP et al (2004) Evaluation of tracheobronchial anomalies in children using low-dose multidetector CT: report of a 13-year-old boy with a tracheal bronchus and recurrent pulmonary infections. Pediatr Pulmonol 38:168-173

2. Siegel MJ (2003) Multiplanar and three-dimensional multidetector row CT of thoracic vessels and airways in the pediatric population. Radiology 229:641-650

3. Salvolini L, Bichi SE, Costarelli L et al (2000) Clinical applications of 2D and 3D CT imaging of the airways - a review. Eur J Radiol 34:9-25

4. Khan MF, Herzog C, Ackermann H et al (2004) Virtual endoscopy of the tracheo-bronchial system: sub-millimeter collimation with the 16-row multidetector scanner. Eur Radiol 14:1400-1405

5. Slovis TL (2003) Children, computed tomography radiation dose, and the as low as reasonably achievable (ALARA) concept. Pediatrics 33:971-972

6. Owens C (2004) Radiology of diffuse interstitial pulmonary disease in children. Eur Radiol Suppl 4:L2-L12

7. Remy J, Remy-Jardin M, Artaud D et al (1998) Multiplanar and three-dimensional reconstruction techniques in CT: impact on chest diseases. Eur Radiol 8:335-351

8. Hoppe H, Dinkel HP, Walder B et al (2004) Grading airway stenosis down to the segmental level using virtual bronchoscopy. Chest 125:704-711

9. Dheda K, Roberts CM, Partridge MR et al (2004) Is virtual bronchoscopy useful for physicians practising in a district general hospital? Postgrad Med J 80:420-423

10. Finkelstein SE, Schrump DS, Nguyen DM et al (2003) Comparative evaluation of super high-resolution CT scan and virtual bronchoscopy for the detection of tracheobronchial malignancies. Chest 124:1834-1840 
11. Wong KS, Wang CR, Hsieh KH (1998) Demonstration of tracheal bronchus associated with tracheal stenosis using direct coronal computed tomography. Pediatr Pulmonol 25:133-135

12. Kosucu P, Ahmetoglu A, Koramaz I et al (2004) Low-dose MDCT and virtual bronchoscopy in pediatric patients with foreign body aspiration. AJR 183:1771-1777

13. Berrocal T, Madrid C, Novo S et al (2004) Congenital anomalies of the tracheobronchial tree, lung, and mediastinum: embryology, radiology, and pathology. Radiographics 24:e17
14. Kussman BD, Geva T, McGowan FX (2004) Cardiovascular causes of airway compression. Paediatr Anaesth 14:60-74

15. Lee KH, Yoon CS, Choe KO et al (2001) Use of imaging for assessing anatomical relationships of tracheobronchial anomalies associated with left pulmonary artery sling. Pediatr Radiol 31:269-278

16. Applegate KE, Dardinger JT, Lieber ML et al (2001) Spiral CT scanning technique in the detection of aspiration of LEGO foreign bodies. Pediatr Radiol 31:836-840 